\title{
BIENESTAR EN TERNEROS LECHALES EN DOS SISTEMAS DE CRIANZA. ESTUDIO DE CASO EN LA CUENCA LECHERA SANTAFESINA
}

\author{
Leva, P.E. ${ }^{1} ;$ García, M.S. ${ }^{1 ;}$ Toffoli, G. ${ }^{1}$; \\ RodrígueZ, A. G. ${ }^{1}$ \& ReY, F. ${ }^{2}$
}

\begin{abstract}
RESUMEN
En el presente trabajo se llevo a cabo un estudio de caso con enfoque cuantitativo. El objetivo de este estudio fue evaluar el bienestar en teneros lechales durante la época invernal bajo dos diferentes sistemas de crianza, que se utilizan en la zona. Los terneros, se alojaron en dos sistemas: en estaca los machos y en jaula las hembras. Los animales recibieron dos tomas diarias de leche (4 1/día), balanceado iniciador y agua ad libitum. Se registraron pesos iníciales y finales. La ganancia de peso se analizó mediante técnicas estadísticas descriptivas. Se tomaron muestra de saliva para determinar cortisol (CS) en cuatro momentos del día. Los datos fueron analizados para detectar variaciones horarias. El CS no mostró fluctuaciones diarias. Durante quince días y día por medio, se registraron las conductas: parado, echado, comiendo y otras. La metodología de observación y registro utilizada fue el muestreo de barrido a intervalos regulares. Los datos de analizaron con la prueba del $\chi^{2}$. El efecto tratamiento fue significativo sobre el comportamiento $(p<0,01)$. Los animales de los dos sistemas gastaron el 50\% del tiempo en la conducta de reposo.

Palabras clave: comportamiento, sistemas de crianza, cortisol en saliva.
\end{abstract}

\section{SUMMARY}

\section{Well-being of suckling calves under two different rearing systems. Case study in the central milk basin of Argentina.}

In this paper we conducted a case study with quantitative approach. The aim of this study was to assess welfare in suckling calves during the winter season under two different rearing systems, which are used in the area. Calves were housed in two systems: a stake in males and females cage. All animals received $4 \mathrm{~L}$ milk per day, in two servings. Water and a commercial starter were offered ad libitum. Initial and final weights were recorded, weight gain was analyzed using descriptive statistical techniques. Saliva samples were taken at four times over the day, to analyze saliva cortisol

1.- Cátedra de Agrometeorología, Facultad de Ciencias Agrarias, Universidad Nacional del Litoral. Kreder 2805. (3080) Esperanza, Santa Fe. Tel/fax: 03496-42-6400; e-mail: pleva@fca.unl.edu.ar 2.- Investigadora del CONICET. Docente de la Facultad de Ciencias Veterinarias, Universidad Nacional del Litoral. Kreder 2805. (3080) Esperanza, Santa Fe.

Manuscrito recibido el 28 de noviembre de 2013 y aceptado para su publicación el 25 de marzo de 2014. 
(SC). Data were analyzed for time variations. Neither daily fluctuations in SC concentrations nor system effects were detected in SC concentrations. Different behaviors: standing, lying, eating and "others" were observed every other day over a fortnight. Scan sampling at regular intervals was utilized. Data were analyzed by a c2 test. Systems effects were observed on behavioral conducts $(\mathrm{p}<0.01)$. Animals in both systems spent $50 \%$ of their time lying.

Key words: behavior, rearing systems, saliva concentrations.

\section{INTRODUCCION}

El sector agropecuario está cambiando vertiginosamente, debido a las tendencias mundiales de globalización, internacionalización de mercados y acuerdos comerciales multinacionales. Uno de los temas emergentes en este escenario es el Bienestar Animal (BA). El bienestar de los animales depende de muchos factores, tales como la sanidad, el alojamiento y el manejo, las interacciones sociales entre animales y la posibilidad de llevar a cabo determinadas pautas de conducta. La conducta puede definirse simplemente como la respuesta de un organismo al ambiente (Carthy, 1969) y el BA está relacionado con el estado del organismo en su intento de adaptarse con ese ambiente (Fraser \& Broom 1993)

El BA en animales recién nacidos es un problema particularmente durante los meses con condiciones ambientales extremas, en donde el ambiente meteorológico juega un papel importante en las primeras semanas de vida. En el caso de los terneros son muy sensibles a la hipotermia cuando los inviernos son fríos y húmedos (Assam et al., 1993). Se registra un incremento del $50 \%$ de muertes en terneros debido a las bajas temperaturas combinadas con lluvia y viento (Berra et al., 1996).Por lo tanto, mejorar el confort de los terneros debe ser una prioridad en los sistemas lecheros, ya que esto podría contribuir a reducir el estrés debido al ambiente (Berra et al., 1996). Las condiciones ambientales en los sistemas de crianza son bastante diferentes a la de un ternero en su vida natural junto a su madre. En los establecimientos comerciales los animales son separados de su madre después del nacimiento y son alimentados con leche o sustitutos que suministran cantidades limitadas de energía y proteína para estimular el desarrollo del rumen y lograr un destete precoz. Por lo tanto, cuando hace demasiado frío o demasiado calor, los animales deben usar energía para mantener su temperatura corporal interna. Esto ocurre a costa de la energía requerida para el crecimiento, por lo que puede tener un efecto negativo sobre el BA (Lagger, 2010).

El estrés tiene un importante rol en la salud de los terneros y es utilizado como un indicador de pobre BA. Los animales pueden sufrir estrés físico: hambre, sed, lesiones, fatiga, condiciones termales extremas, o estrés psicológico: restricción de los movimientos, practicas de manejo, cambios imprevisto (Grandin, 1997). Otro aspecto a considerar, es la ritmicidad de la temperatura del cuerpo, de la respiración y de las hormonas que son procesos biológico importantes y marcadores convenientes y confiables del funcionamiento del reloj 